\title{
Selective corticostriatal plasticity during acquisition of an auditory discrimination task
}

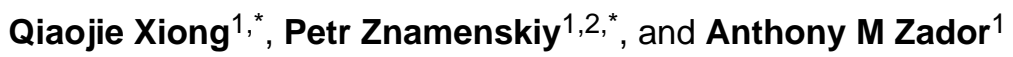 \\ ${ }^{1}$ Cold Spring Harbor Laboratory, 1 Bungtown Road, Cold Spring Harbor, New York 11724, USA \\ ${ }^{2}$ Watson School of Biological Sciences, Cold Spring Harbor Laboratory, 1 Bungtown Road, Cold \\ Spring Harbor, New York 11724, USA
}

\begin{abstract}
Perceptual decisions are based on the activity of sensory cortical neurons, but how organisms learn to transform this activity into appropriate actions remains unknown. Projections from the auditory cortex to the auditory striatum carry information that drives decisions in an auditory frequency discrimination task ${ }^{1}$. To assess the role of these projections in learning, we developed a Channelrhodopsin-2-based assay to selectively probe for synaptic plasticity associated with corticostriatal neurons representing different frequencies. Here we report that learning this auditory discrimination preferentially potentiates corticostriatal synapses from neurons representing either high or low frequencies, depending on reward contingencies. We observed frequency-dependent corticostriatal potentiation in vivo over the course of training, and in vitro in striatal brain slices. Our findings suggest a model in which the corticostriatal synapses made by neurons tuned to different features of the sound are selectively potentiated to enable the learned transformation of sound into action.
\end{abstract}

\begin{abstract}
Animals use sensory information to guide their behavior. The neural mechanisms underlying the transformation of sensory responses into motor commands have been studied extensively using a two alternative forced choice (2AFC) paradigm, in which subjects are trained to make a binary decision and indicate their choice by performing one of two actions. Defined brain areas have been implicated in the circuit performing this transformation in primates ${ }^{2,3}$ and rodents ${ }^{1,4-10}$.
\end{abstract}

Striatal plasticity has been implicated in reinforcement learning ${ }^{11,12}$, specifically at corticostriatal inputs ${ }^{13,14}$, but the site or sites of plasticity engaged when animals learn to make appropriate decisions about sensory stimuli are not well established. We previously

\footnotetext{
Users may view, print, copy, and download text and data-mine the content in such documents, for the purposes of academic research, subject always to the full Conditions of use:http://www.nature.com/authors/editorial_policies/license.html\#terms

Correspondence to: Anthony M Zador, Cold Spring Harbor Laboratory, 1 Bungtown Road, Cold Spring Harbor, NY 11724, zador@cshl.edu, Tel 516-367-6950, Fax: 516-367-8866.

These authors contributed equally to the work

Competing financial interests: The authors declare no competing financial interests.

Contributions

Q.X., P.Z., and A.M.Z designed the experiments; Q.X. performed the experiments; Q.X., P.Z., and A.M.Z performed the data analysis and wrote the manuscript.
} 
found that neurons in the primary auditory cortex projecting to the auditory striatum drive decisions in a $2 \mathrm{AFC}$ auditory task ${ }^{1}$ in which rats learn to associate the frequency of a complex auditory stimulus with either a left or right reward port (Fig. 1a\&b). We hypothesized that plasticity of auditory corticostriatal connections encodes the association between frequency and the rewarded response.

To test this hypothesis, we developed a novel in vivo recording paradigm with which we could monitor the strength of corticostriatal synapses, in a way that did not depend on the activity of cortical neurons. We used this paradigm to measure synaptic strength in single animals over multiple behavioral sessions during the course of learning. We first injected an adeno-associated virus expressing Channelrhodopsin-2 (AAV-ChR2-Venus) into the left primary auditory cortex. This resulted in widespread expression of ChR2 in different cell types in the auditory cortex, including corticostriatal neurons and their axons in the striatum (Extended Data 1). We next implanted bundles of optical fibers and tetrodes into the left auditory striatum (Fig. 1c). Brief pulses of blue light delivered through the optical fiber excited the corticostriatal axons and elicited excitatory postsynaptic responses in the striatum (Fig. 1d). Because the striatum, like the CA1 region of the hippocampus, lacks recurrent excitatory connections, we reasoned that this in vivo ChR2-evoked local field potential response (ChR2-LFP) could serve as a measure of the strength of the corticostriatal synaptic connectivity ${ }^{15}$. The ChR2-LFP had a stereotypic waveform consisting of an early and a late component (Extended Data 2a). Local pharmacological blockade of excitatory but not inhibitory transmission diminished the late component, indicating that it was mainly mediated by currents elicited by glutamatergic release from corticostriatal terminals (Fig. 1d, Extended Data 2c). The early component was resistant to all blockers including tetrodoxin, suggesting that it is driven directly by light-evoked ChR2 currents in corticostriatal axons. The early component was not observed in the absence of ChR2 (Extended Data 3), indicating that it was not due to a photoelectric artifact, and its amplitude increased with increasing photostimulation (Extended Data 4). In subsequent analyses we normalized the ChR2-LFP to the amplitude of the early component (Extended Data 2a) to correct for fluctuations in the number of ChR2-expressing fibers recruited, and then used the initial slope of the second component as a measure of corticostriatal synaptic efficacy (Fig. 1d \& Extended Data 2b). This metric was robust to changes in light intensity and was proportional to the intracellular EPSC, indicating that it was a good measure of synaptic strength (Extended Data 4).

We used the ChR2-LFP to assess changes in the strength of corticostriatal synapses over the course of training in the cloud-of-tones task. After establishing a stable baseline over several days in naïve rats, we measured the ChR2-LFP after each training session. We used the tone-evoked multiunit responses recorded prior to training to estimate the frequency tuning at each site (see Methods). At some recording sites, corticostriatal synaptic efficacy increased as soon as the animal started to learn the task, and continued to increase in subsequent training sessions (Fig. 2a). Synaptic efficacy at such sites thus reflected behavioral performance over the course of training. At other sites, however, corticostriatal efficacy remained unchanged over the course of training (Fig. 2b). We found that potentiation was restricted to sites tuned to low-frequency $(<14 \mathrm{kHz}$, the center frequency 
used in the task) sounds (mean potentiation: 30\%; $\mathrm{n}=17, p=0.002$, signed-rank test, Fig. $2 \mathrm{c}$ ), whereas sites tuned to high-frequency $(>14 \mathrm{kHz})$ sounds showed no significant change (mean change: $-3 \% ; \mathrm{n}=6, p=0.58$, signed-rank test, Fig. 2c.). Notably, all animals in this cohort were trained to associate low frequency sounds with rightward choices (LowRight), and all recordings were performed in the left striatum. Hence, low frequencies were always associated with choices contralateral to the recording hemisphere. Our results therefore suggested that task training selectively enhances the strength of corticostriatal synapses only when the stimuli they encode are associated with contralateral choices (Fig. 2d).

The observed potentiation depended strongly on the frequency tuning of the recording site, suggesting that corticostriatal plasticity encodes the association of specific frequencies with rewarded actions. However, since the striatum has been widely implicated in motor learning ${ }^{14}$, we sought to rule out this and other alternative causes of plasticity unrelated to auditory discrimination. We trained animals to perform a simple $2 \mathrm{AFC}$ visual task, relying on the same sequence of movements, and monitored the strength of auditory corticostriatal synapses during learning (Fig. 3a). There was no significant change in ChR2-LFP in the auditory striatum during visual task training (mean change: $-17 \%, \mathrm{n}=12, p=0.13$, signedrank test), and there was no correlation between potentiation and the preferred frequency at the recording site ( $\mathrm{n}=12, p=0.19$; Fig. $3 \mathrm{~d}$ ). However, corticostriatal inputs at these same recording sites were potentiated when the animals subsequently learned the auditory cloudof-tones task (mean potentiation: 36\%, $\mathrm{n}=6, p=0.03$, signed-rank test; Fig. 3b\&c). We therefore conclude that the selective potentiation of auditory corticostriatal synaptic strength is specific to the acquisition of the auditory task.

The preferential potentiation in vivo of striatal sites tuned to low frequencies suggested that the pattern of potentiation might be spatially organized within the striatum. We therefore developed an in vitro brain slice preparation to examine this possibility. We first characterized the tonotopic organization of the auditory corticostriatal projection by injecting AAVs encoding either red or green fluorescent proteins at two different positions along the auditory cortical tonotopic axis. Cortical axons terminated in the striatum in distinct bands, with cortical projections tuned to high frequency sounds terminating more laterally in the auditory striatum and projections tuned to low frequency sounds more medially (Fig. 4a \& Extended Data 5). We next developed a protocol to assess the gradient of corticostriatal potentiation along the tonotopic axis, by recording ChR2-LFPs in coronal slices that preserve striatal tonotopy (Fig. 4b, see Methods). These recordings targeted left striatum, contralateral to the reward direction associated with low-frequency stimuli (LowRight; $\mathrm{n}=7$ rats). For consistency across experiments, we used only a single slice from each animal, selected on the basis of striatal and hippocampal landmarks (see Methods). ChR2-LFPs in these slices showed a stereotyped waveform similar to that observed in vivo, and pharmacological dissection confirmed that the late component of the response was mediated mainly by AMPA-type glutamate receptors (Fig. 4c). Simultaneous extracellular and intracellular recording indicated that the ChR2-LFP was a faithful measure of synaptic strength (Fig. 4d \&e, top). As expected, the normalization corrected for changes in ChR2LFP induced by recruiting more presynaptic fibers (Fig. 4e, bottom), but did not obscure true changes in synaptic strength induced by changes in release probability (Fig. 4d, bottom). 
To measure gradients in synaptic strength along the tonotopic axis induced by training, in each slice we recorded the ChR2-LFP at between 8 and 16 sites $(12.1 \pm 2.1)$. Naïve rats showed no systematic difference in the strength of cortical input along the striatal tonotopic axis (Extended Data 6). In contrast, in rats trained to associate low frequencies with rightward choices, the evoked corticostriatal response was strongest at medial (low frequency) sites and decreased laterally (Fig. $4 \mathrm{f} \& \mathrm{~g}$ ). This confirmed our observations in vivo and was consistent with the association to contralateral rewards. Thus, the degree of corticostriatal synaptic potentiation induced by learning depended systematically on the position along the striatal tonotopic axis.

If the gradient of potentiation along the striatal tonotopic axis encodes the association between frequency and choice direction, then animals trained to make the opposite association should have a gradient of opposite sign. To test this we trained a new cohort of animals to associate low frequencies with leftward choices (LowLeft; $n=7$ rats). As predicted, the gradient in these animals was of similar magnitude but opposite in sign (Fig. $4 \mathrm{~g})$. There was no difference between these two training groups in ChR2-LFP across the orthogonal (dorsoventral) axis ( $p=0.22$, paired $t$-test; Extended Data 7). Thus the spatial gradient of corticostriatal potentiation induced by learning along the tonotopic axis depends on the training contingencies to which the animal is subjected.

Finally, we wondered whether the direction of the stimulus-response association could be inferred based on the sign of the ChR2-LFP gradient in individual animals. Remarkably, the training history (LowRight vs. LowLeft) of every rat (14/14) could be correctly inferred from the sign of gradient in a single slice (binomial test $p=0.00006$, Fig. $4 \mathrm{~h}$ ). The correlation between synaptic strength and tonotopic position reached statistical significance $(p<0.05)$ in 6 of 14 slices. Thus post-mortem study of corticostriatal efficacy can reliably reveal the training history of individual subjects.

Our results suggest a simple model of how the specific pattern of corticostriatal potentiation we observed might mediate task acquisition. In the LowRight task, training selectively potentiated corticostriatal synapses tuned to low frequencies between the left auditory cortex and the left auditory striatum (Extended Data 8). Thus in behaving animals, low frequency tones would trigger stronger activation in the left auditory striatum and direct the animal to the right (contralateral) response port, possibly through the action of direct pathway medium spiny neurons ${ }^{10}$ that project ipsilaterally to the left substantia nigra pars reticulata and in turn to the superior colliculus ${ }^{16}$. On the other hand, in LowLeft trained animals, potentiation would cause the same stimulus to trigger stronger activation in right auditory striatum and direct the animal to the left response port. Although this model ignores much of the complexity of striatal circuitry, it offers a simple framework for understanding our results.

Previous work has demonstrated synaptic ${ }^{17-20}$ or receptive field ${ }^{21-26}$ changes induced by learning. Our results identify a locus of synaptic plasticity during the acquisition of a sensory discrimination task. We focused on auditory frequency discrimination, which allowed us to exploit the spatial organization of auditory corticostriatal connections to relate the tuning of cortical neurons to plasticity. Training selectively enhanced the strength of 
cortical inputs to establish an orderly gradient of corticostriatal synaptic strength across the striatal tonotopic axis.

The strengthening of a subset of connections, selected from a rich sensory representation, is reminiscent of several powerful models of learning ${ }^{27-29}$. In these models, even difficult nonlinear classification can be achieved by combining a high-dimensional representation of the stimulus—-such as is found in the auditory cortex ${ }^{30}$ — through simple reinforcement learning rules. We speculate that selective strengthening of appropriate corticostriatal synapses would allow animals to categorize a wide range of sensory stimuli-even those that are not mapped topographically in the striatum—and may reflect a general mechanism through which sensory representations guide the selection of motor responses.

\section{Materials and Methods}

\section{Animals and viruses}

Animal procedures were approved by the Cold Spring Harbor Laboratory Animal Care and Use Committee and carried out in accordance with National Institutes of Health standards. AAV-CAGGS-ChR2-Venus serotype 2/9 was packaged by the University of Pennsylvania Vector Core.

Long Evans male rats (Taconic Farm) were anaesthetized with a mixture of ketamine (50 $\mathrm{mg} \mathrm{kg}-1)$ and medetomidine $\left(0.2 \mathrm{mg} \mathrm{kg}^{-1}\right)$, and injected with virus at 3-4 weeks old in the left auditory cortex. To cover most of the area and layers of the primary auditory cortex, 3-4 injections were made perpendicularly to brain surface at 1,2 , and $3 \mathrm{~mm}$ caudal to the temporoparietal suture, and $1 \mathrm{~mm}$ from the ventral edge. Each injection was made at three depths (500, 750 and $1000 \mu \mathrm{m})$, expelling approximately $200 \mathrm{nl}$ of virus at each depth.

\section{Behavioural training}

Rats were placed on a water deprivation schedule and trained to perform an auditory $2 \mathrm{AFC}$ task in a single-walled sound-attenuating training chamber as described previously ${ }^{1}$. Briefly, freely moving rats were trained to initiate a trial by poking into the center port of a threeport operant chamber, which triggered the presentation of a stimulus. Subjects then selected the left or right goal port. Correct responses were rewarded with water. The cloud-of-tones stimulus consisted of a stream of 30-ms overlapping pure tones presented at $100 \mathrm{~Hz}$. The stream of tones continued until the rat withdrew from the center port. Eighteen possible tone frequencies were logarithmically spaced from 5 to $40 \mathrm{kHz}$. For each trial either the low stimulus ( 5 to $10 \mathrm{kHz}$ ) or high stimulus ( 20 to $40 \mathrm{kHz}$ ) was selected as the target stimulus, and the rats were trained to report low or high by choosing the correct side of port for water reward. In LowLeft task, the rats were required to go to the left goal port for water reward when the low stimulus was presented, and to the right goal port when high stimulus was presented. In LowRight task, the rats were required to go to the right goal port when the low stimulus was presented, and to the left goal port when the high stimulus was presented.

The pre-stimulus delay was drawn from exponential distribution with a mean of $300 \mathrm{~ms}$. Early withdrawal from the center port before the onset of stimuli terminated the trial and a new trial was started. To complete a trial after exiting the center port, the animals were 
allowed up to 3 seconds to select a reward port. Typically, they made their choice within 300-700 ms. Error trials, where the rats reported to the wrong goal port after the presentation of the stimulus, were penalized with a 4 second time-out.

The intensity of individual tones was constant during each trial. To discourage rats from using loudness differences in discrimination, tone intensity was randomly selected on each trial from a uniform distribution between 45 and $75 \mathrm{~dB}$ (SPL) during training.

Implanted rats were water deprived and given free water for 1 hour every day before ChR2LFP recording. These sessions were used to record baseline ChR2-LFP responses and defined as naïve sessions. Once a stable baseline was achieved, we began training subjects to perform the cloud-of-tones task. To introduce the subjects to the task structure, they were first trained ("direct mode") to poke at the center port, which triggered the presentation of a stimulus and elicited water delivery from the corresponding goal port. Direct mode training was continued until a subject completed at least 150 trials in a single session (usually the first or second session). In subsequent sessions, defined as "session 1" in Figs. 2 and 3, the animal was trained on the "full task," in which water was delivered only if the subject poked the correct goal port. For control subjects used in Fig. 3, subjects were trained in the direct mode with visual stimuli prior to introducing the full visual task, and then trained on the full auditory task.

\section{Tetrode recording and optogenetics}

Custom tetrode and optic fiber arrays were assembled as described previously ${ }^{1}$. Each array carried 6 individually movable microdrives. Each microdrive consisted of one tetrode (4 polyimide-coated nichrome; wire diameter $12.7 \mu \mathrm{m}$; Kanthal Palm Coast) twisted together and gold-plated to an impedance of $0.3-0.5 \mathrm{M} \Omega$ at $1 \mathrm{kHz})$ and one optic fiber $(62.5 \mu \mathrm{m}$ diameter with a 50- $\mu \mathrm{m}$ core; Polymicro Technologies). The tetrode and fiber on the same microdrive were glued together, with the tips approximately $100 \mu \mathrm{m}$ from each other.

To implant the tetrode/fiber array, rats were anaesthetized with a mixture of ketamine (50 $\left.\mathrm{mg} \mathrm{kg}^{-1}\right)$ and medetomidine $\left(0.2 \mathrm{mg} \mathrm{kg}^{-1}\right)$ and placed in a stereotaxic apparatus. A craniotomy was made above the target area $(2.5$ to $3.5 \mathrm{~mm}$ from Bregma and $4 \mathrm{~mm}$ to $6 \mathrm{~mm}$ lateral from the midline). All rats were implanted in left hemisphere. The array was fixed in place with dental acrylic, and the tetrodes were lowered down to auditory striatum ( 3 to 5 $\mathrm{mm}$ from pia).

Electrical signals in auditory striatum were recorded using Neuralynx Cheetah 32-channel system and cheetah data acquisition software. For action potential recording, signals were filtered 600 to $6000 \mathrm{~Hz}$. For local field potential recording, signals were filtered 10 to 9000 $\mathrm{Hz}$. The rise time of the ChR2-LFP is relatively fast, so to preserve its dynamics we sought to stay as close to the raw data as possible. We re-analyzed the data in Fig 2a using two other choices of offline filter (median, $660 \mathrm{msec}$ window \& Butterworth lowpass 1-800 Hz). As shown in supplementary Extended Data 9a\&b, although filtering does affect the details of the ChR2-LFP shape (especially the earliest component), the results are qualitatively unchanged (Extended Data 9c). 
To determine the preferred sound frequency of recording sites, pure tones spanning from 1 $\mathrm{kHz}$ to $64 \mathrm{kHz}$ were presented to rats before the start of behavioral training in a soundproof chamber for $100 \mathrm{~ms}$ every $2 \mathrm{~s}$, in a random order at 30,50 or $70 \mathrm{~dB}$ (SPL) ${ }^{1}$. The multi-unit baseline-subtracted firing rate in a window 5 to $55 \mathrm{~ms}$ after sound onset was compared with that in a window 0 to $50 \mathrm{~ms}$ before sound onset; only sites that significantly responded to sound were included. Firing rates in the window 5 to $55 \mathrm{~ms}$ after sound onset were computed for each frequency at $70 \mathrm{~dB}$, and the peak of the resulting tuning curve was selected as the preferred frequency.

For ChR2-LFP recording, $473 \mathrm{~nm}$ laser light was delivered through an FC/PC patch cord using a FiberPort Collimator (Thor Labs) to each implanted fiber individually. ChR2-LFP was recorded immediately after each training session. The laser power out of the patch cord was measured and adjusted to elicit an LFP with clear early and delayed components at each recording site (1-10 mW). For individual recording sites, laser power was adjusted slightly between days to maintain the early, presynaptic component of the LFP response at a consistent level. Each light pulse was $100 \mu$ s in duration, presented at $1 \mathrm{~Hz}$, and each recording was an average of approximately 100 trials.

\section{In vivo pharmacology}

To dissect the components of ChR2-LFP in vivo, rats were anaesthetized and placed in a stereotaxic apparatus. A single tetrode/fiber bundle was placed on a motorized manipulator (Sutter Instrument Company) and the tips of tetrode/fiber were guided to auditory striatum. Glass pipettes were used to deliver chemicals into auditory striatum. The pipettes filled with desired chemicals were carefully moved to penetrate through cortex and placed with the tips close to auditory striatum. Air pressure was slowly applied to inject the chemicals into tissue through a syringe that was connected to the pipette.

\section{Slice recording}

Virus-injected and trained rats were anesthetized and decapitated, and the brains were transferred to a chilled cutting solution composed of (in $\mathrm{mM}$ ) 110 choline chloride, 25 $\mathrm{NaHCO}_{3}, 25 \mathrm{D}$-glucose, 11.6 sodium ascorbate, $7 \mathrm{MgCl}_{2}, 3.1$ sodium pyruvate, $2.5 \mathrm{KCl}$, $1.25 \mathrm{NaH}_{2} \mathrm{PO}_{4}$ and $0.5 \mathrm{CaCl}_{2}$. Coronal slices $(350 \mu \mathrm{m})$ were cut and transferred to artificial cerebrospinal fluid (ACSF) containing (in $\mathrm{mM}$ ) $127 \mathrm{NaCl}, 25 \mathrm{NaHCO}_{3}, 25 \mathrm{D}$-glucose, 2.5 $\mathrm{KCl}, 4 \mathrm{MgCl}_{2}, 1 \mathrm{CaCl}_{2}$ and $1.25 \mathrm{NaH}_{2} \mathrm{PO}_{4}$, aerated with $95 \% \mathrm{O}_{2} 5 \% \mathrm{CO}_{2}$.

To ensure maximal alignment across animals, only a single slice ( $350 \mu \mathrm{m}$ thickness, between $2.5 \mathrm{~mm}$ and $2.9 \mathrm{~mm}$ from Bregma) per animal was used. Slices were incubated at $34{ }^{\circ} \mathrm{C}$ for 15-20 min and then kept at room temperature $\left(22^{\circ} \mathrm{C}\right)$ during the experiments. Local field potentials were recorded using Axopatch 200B amplifiers (Axons Instruments, Molecular Devices).

We delivered light pulses through a light guide microscope illumination system (Lumen Dynamics) modified to accept a blue laser (473 nm, Lasermate Group, CA, USA) in place of the lamp. The laser beam was focused onto the sample through the $60 \mathrm{X}$ objective during recordings, with illumination field of 350- $\mu$ m diameter. Each light pulse was $500 \mu \mathrm{s}$ at $1 \mathrm{~Hz}$, 
and each recording was an average of approximately 10 trials. Laser power was adjusted from site to site to maintain a similar level of axonal stimulation as judged by the amplitude of the early, presynaptic component of the ChR2-LFP response. To minimize the contribution of rundown on the estimation of the plasticity gradient within the striatal slice, recording locations were selected in randomly for each slice.

\section{Data analysis}

All data were analyzed in MATLAB.

Behavior analysis only included completed trials. The percentage of correct trials for each animal in each session was computed using the last 200 trials of that session, unless the number of trials was less than 300 , in which case only the last 100 trials were used.

Each measurement of in vivo ChR2-LFP was from a trace obtained by averaging across 70100 trials (The slope measured from averaged trace is not different from the averaged slope from those of single traces, Extended Data 10). Each average trace was normalized to the peak of the first component (around the time window between $0.5 \mathrm{~ms}$ to $1.2 \mathrm{~ms}$ after light stimulation, Extended Data 2a), and the LFP slope was estimated by a linear regression fit of the rising phase of the second component (Extended Data 2b, same time window was used for each recording site across sessions, the time window from site to site varied and was adjusted by eye for each site, ranged from $1.6 \mathrm{~ms}$ to $5 \mathrm{~ms}$ after light stimulation). The ChR2LFP slope for each recording site across sessions was used as a measure of synaptic strength in subsequent analyses (Fig. 2a\&b, Fig. 3b). The absolute change of the ChR2-LFP slope was used in Fig. $2 \mathrm{~d} \& 3 \mathrm{~d}$. In population analyses, normalized synaptic strength for each site was obtained by dividing the LFP slope values from all sessions by the mean of the ChR2LFP slope values from naïve sessions (Fig. 2c \& 3c).

For quantification of the ChR2-LFP in slice recording, the ChR2-LFP slope at each recording site was obtained in a manner similar to that in vivo: each averaged trace was normalized to the peak of the first component (around the time window between $1.5 \mathrm{~ms}$ to 4 ms after light stimulation), and the ChR2-LFP slope was estimated by a linear regression fit of the rising phase of the second component (around the time window between $5 \mathrm{~ms}$ to $8 \mathrm{~ms}$ after light stimulation, adjusted by eye for each slice). For each slice, the ChR2-LFP slopes across sites were rescaled from 0 to 1 , with the smallest ChR2-LFP set to zero and the largest to 1 . All recorded brain slices were aligned to a consensus brain slice. The positions of recording sites were measured from the aligned brain slices. Data from all the slices were pooled together for plotting the summary plot and for the quantitative analysis. 


\section{Extended Data}

a

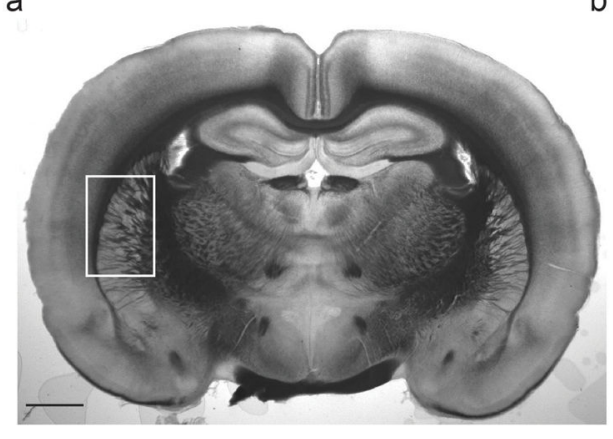

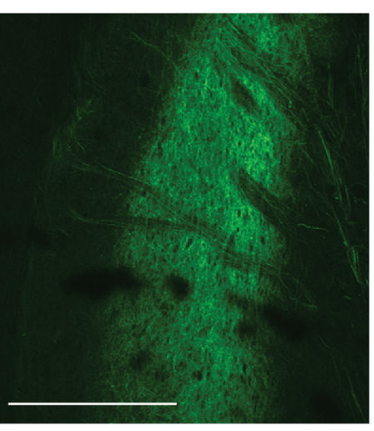

\section{Extended Data 1.}

Corticostriatal projections from auditory cortex to striatum. (a) Coronal view for the location in the striatum that receives auditory cortical inputs. (b) Confocal image of auditory cortical axon terminals expressing ChR2-Venus in the striatum. Scale bars: $2 \mathrm{~mm}$. 
a

Raw ChR2-LFP traces Normalized ChR2-LFP traces

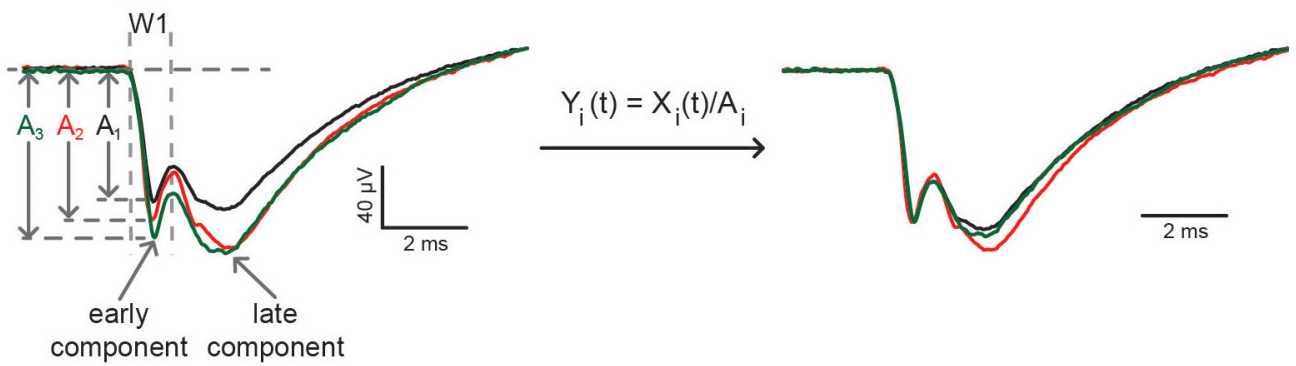

b

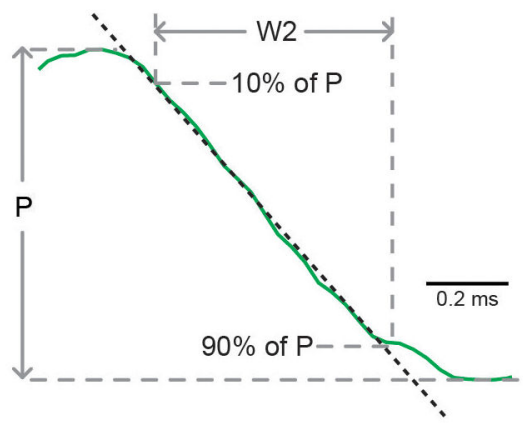

C
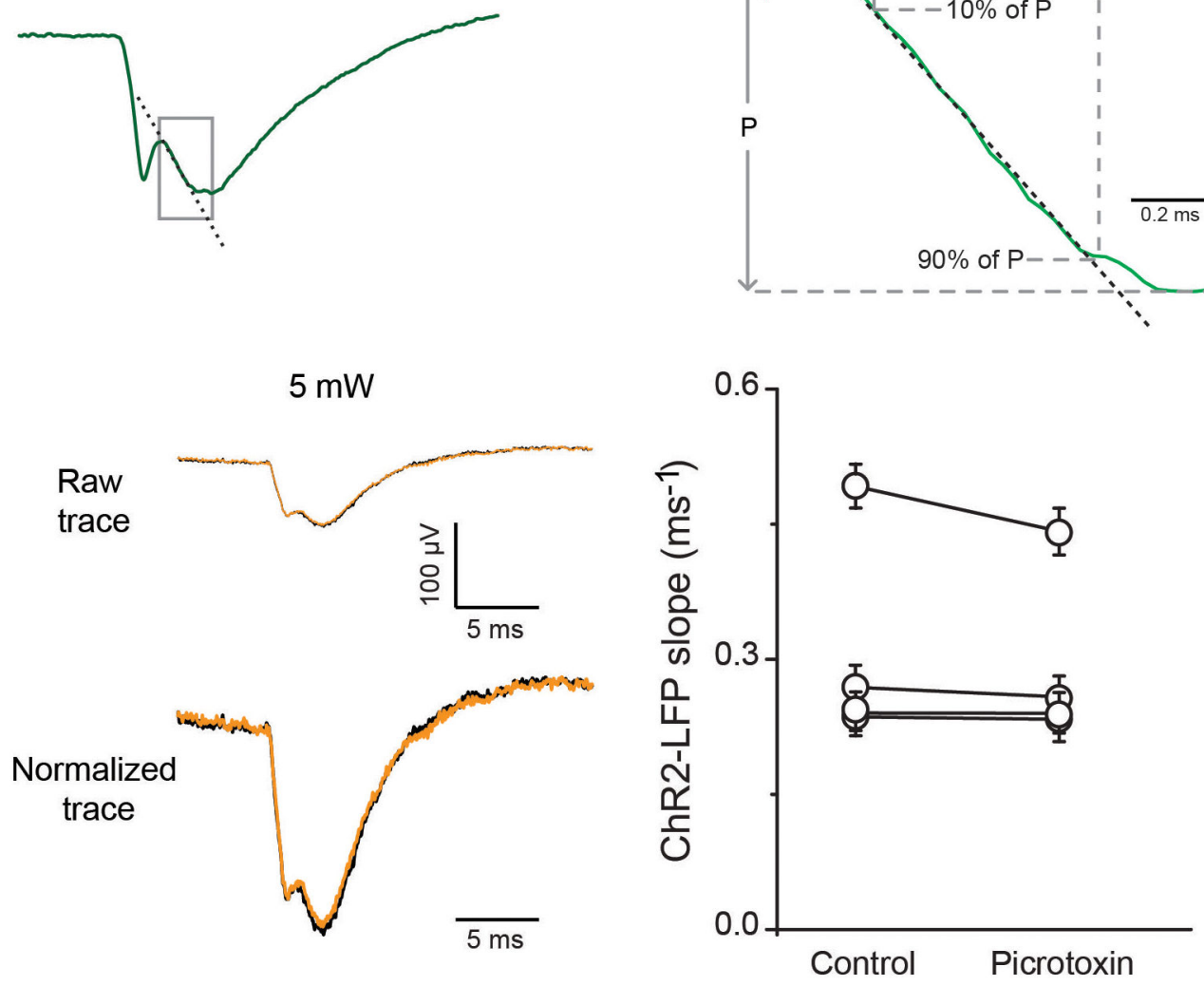

Extended Data 2.

Slope measurement for ChR2-LFP and GABAergic synaptic transmission does not contribute to the CHR2-LFP slope in vivo. (a) Raw ChR2-LFP traces (left panel) were normalized to the amplitude of their corresponding early component $\left(A_{i}\right)$. The normalization factor $A_{i}$ was determined as the peak of the raw trace in the time window (W1) between 0.5 $\mathrm{ms}$ to $1.2 \mathrm{~ms}$ after light stimulation onset. (b) The rising phase of the late component of ChR2-LFP (in a time window W2 defined by rise from $10 \%$ to $90 \%$ of the peak P) was fit linearly, and the slope of the fit was used for the quantification of ChR2-LFP. (c) left panels: ChR2-LFP before (black traces) and after (orange traces) picrotoxin application (20 mM, 5 ul). Raw traces are averaged traces from 60-80 trials at each condition (upper row).

Normalized traces are raw traces normalized to their peaks of first components (as illustrated 
in a). Right panel: slopes measured from normalized traces in control and picrotoxin conditions for each recording before and after picrotoxin application $(p=0.8$, paired signedrank test). Data are presented as mean \pm s.e.m.

a
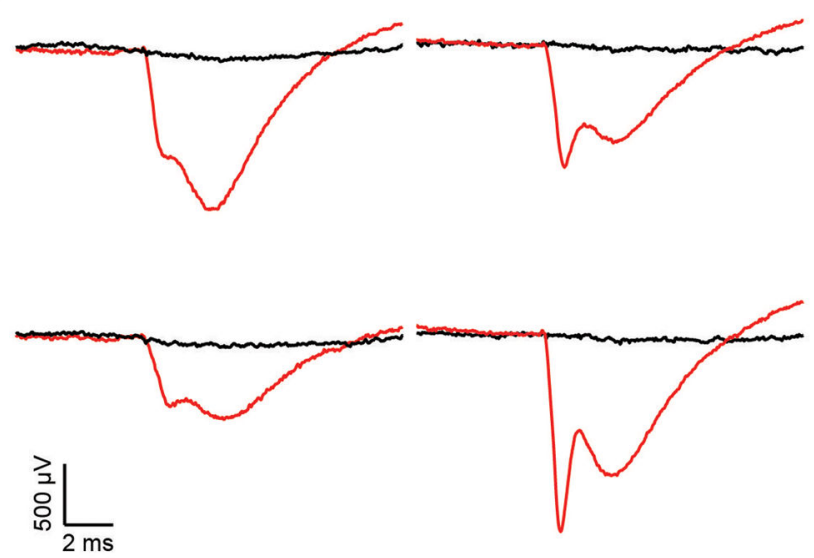

b

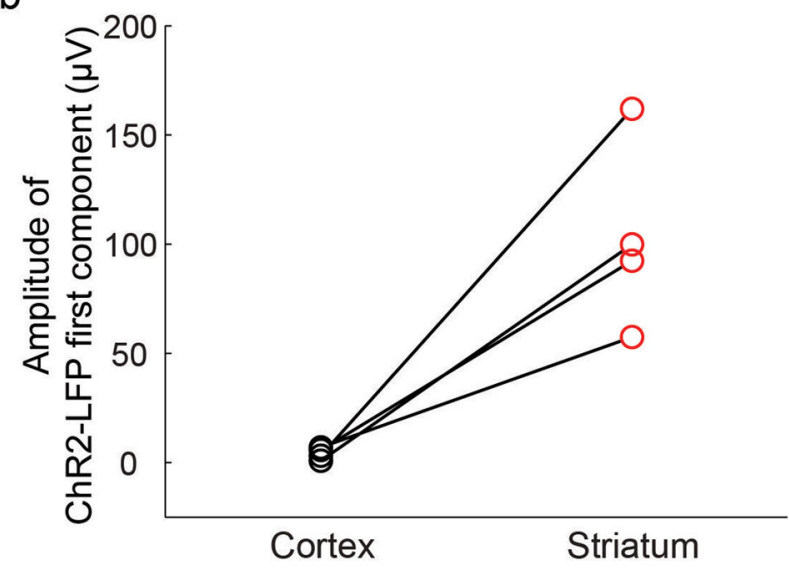

Extended Data 3.

ChR2-LFP depends on presence of ChR2-expressing axons. To rule out the possibility that the TTX-insensitive component of the light-evoked response resulted from a photoelectric or other artifact, rather than from ChR2-evoked currents, we assessed light-evoked responses in brain regions that did not express ChR2. (a) Four independent recordings in the auditory striatum (red traces) which receives auditory cortical input (ChR2-expressing axons are present), and the overlying somatosensory cortex (black traces) which lacks auditory cortical input (ChR2-expressing axons are absent). Each pair of recordings is from the same tetrode/ fiber bundle. The recordings indicate that the light artifact is negligible under our conditions. (b) Comparison of the first component amplitude from each recording pair.

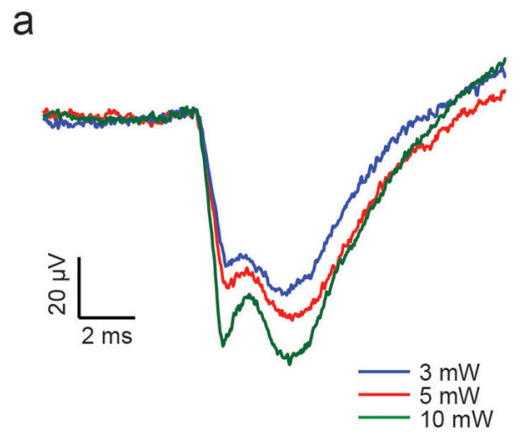

b

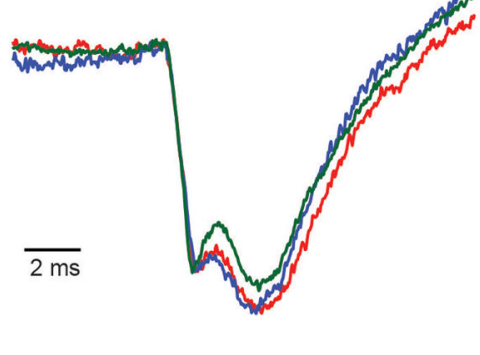

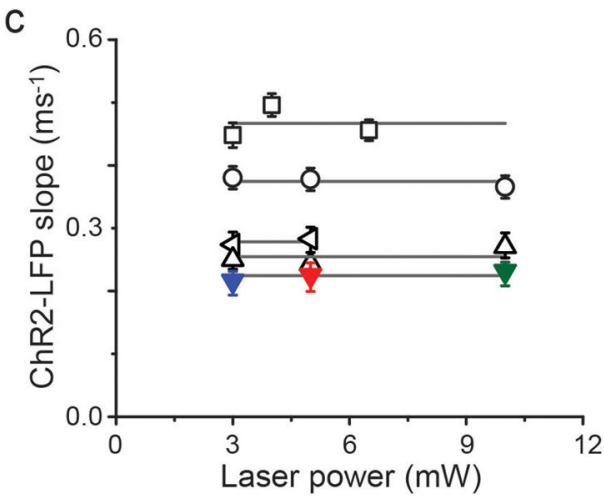

Extended Data 4.

Normalization procedure corrects for variation in light power in vivo (for in vitro data see Fig. 4 d\&e). (a) Example of ChR2-LFP recorded at different light levels. (b) Normalized ChR2-LFP the same example in a. (c) Slopes from 5 example recordings across 1-10 $\mathrm{mW}$ light level range (colored symbols are examples shown in $\mathbf{a} \& \mathbf{b}$ ). Grey lines are drawn from the mean values of each group. Together with the data shown in Fig. 4e, the normalization 
procedure thus minimizes fluctuations in the response arising from artifactual changes in the number of recruited fibers, but preserves changes arising from actual increases or decreases in synaptic efficacy.

a

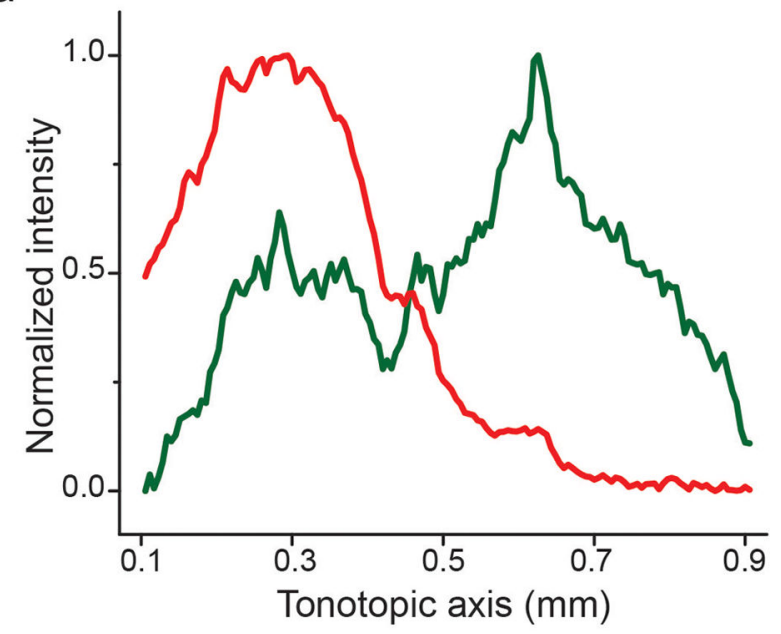

b

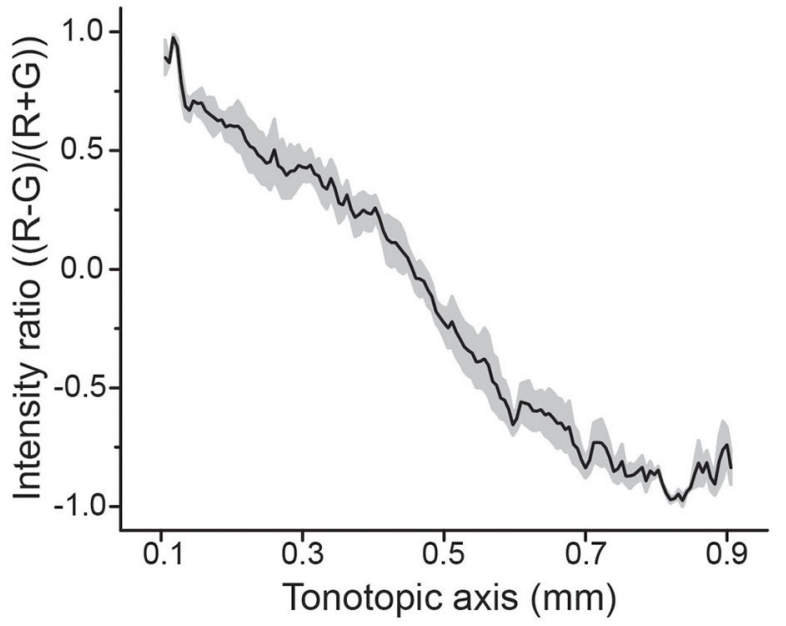

Extended Data 5.

Quantification of corticostriatal projection topography. (a) Normalized red and green fluorescence intensities measured across the tonotopic axis from image shown in Figure 4a. (b) Mean red/green intensity ratio across the tonotopic axis, $\mathrm{n}=3$ sections from 2 rats. Shading, s.e.m. 


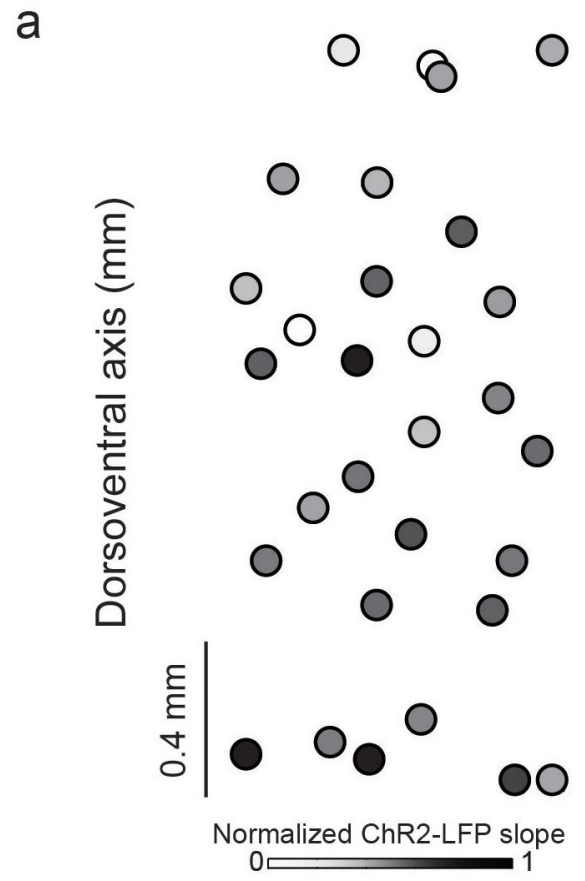

b

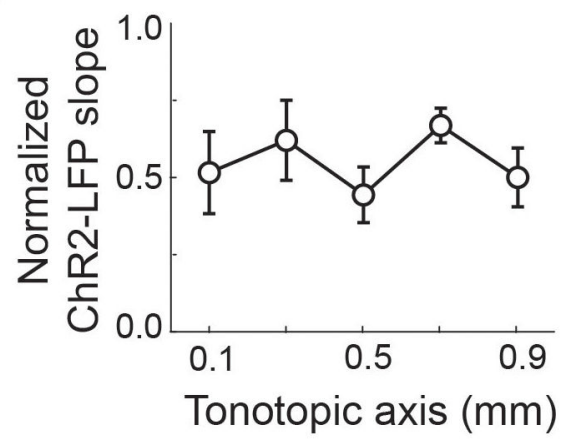

Extended Data 6.

ChR2-LFP slope does nor vary systematically across tonotopic axis in naïve rats. (a) ChR2LFP slope map from 3 striatal slices ( $n=3$ rats). (b) Quantification of ChR2-LFP slope across tonotopic axis. Data are presented as mean \pm s.e.m. 
a

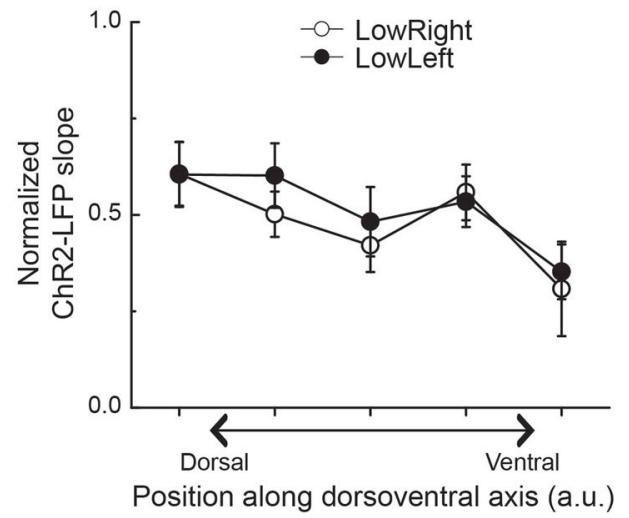

b

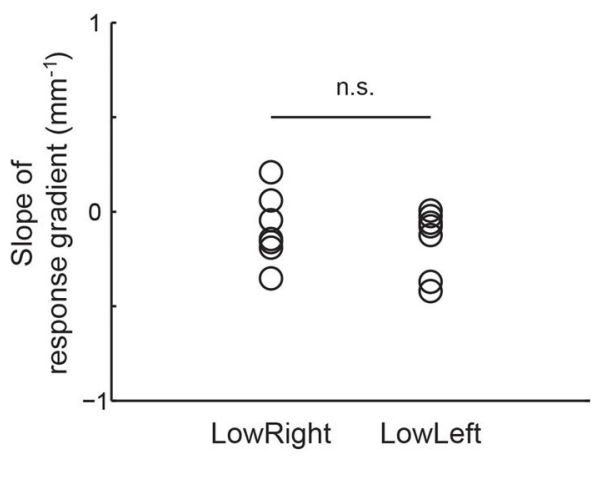

Extended Data 7.

Gradient of ChR2-LFP across the dorsoventral (non-tonotopic) axis showed no difference between the two training groups. (a) Averaged ChR2-LFP slopes with position along tonotopic axis for LowRight and LowLeft (7 rats from each group). (b) Individual gradients of ChR2-LFP across dorsoventral aixs from LowRight and LowLeft groups $(p=0.22$, paired $t$-test).

a

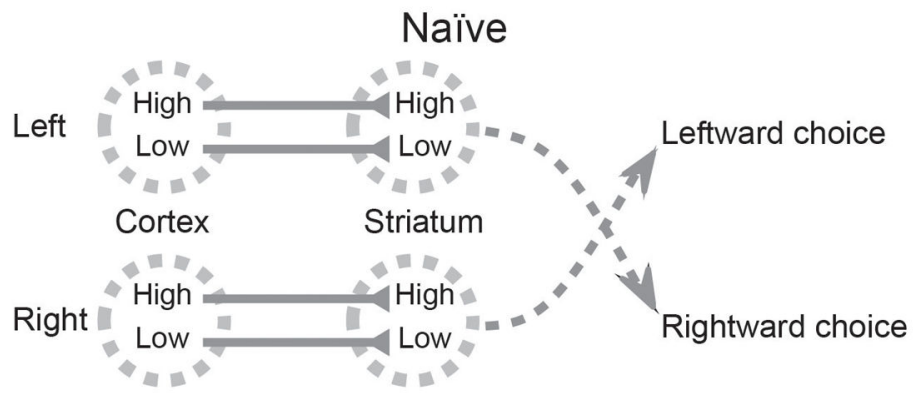

b

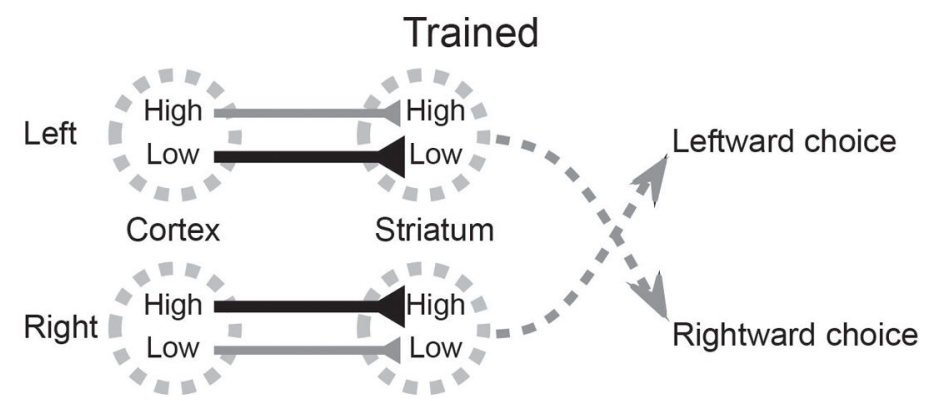

Extended Data 8.

Model showing how corticostriatal potentiation could mediate task acquisition. (a) In the naïve rat, the strength of corticostriatal connections does not depend on their frequency preference. (b) Training to associate low stimuli with rightward choices and high stimuli with leftward choices (LowRight) selectively potentiates corticostriatal synapses tuned to 
low frequencies in the left hemisphere and corticostriatal synapses tuned to high frequencies in the right hemisphere. Thus in the trained rat, low stimuli drive rightward choices and high stimuli drive leftward choices.

a

Unfiltered

Median filtered (660 ms window)

Butterworth lowpass $(800 \mathrm{~Hz})$
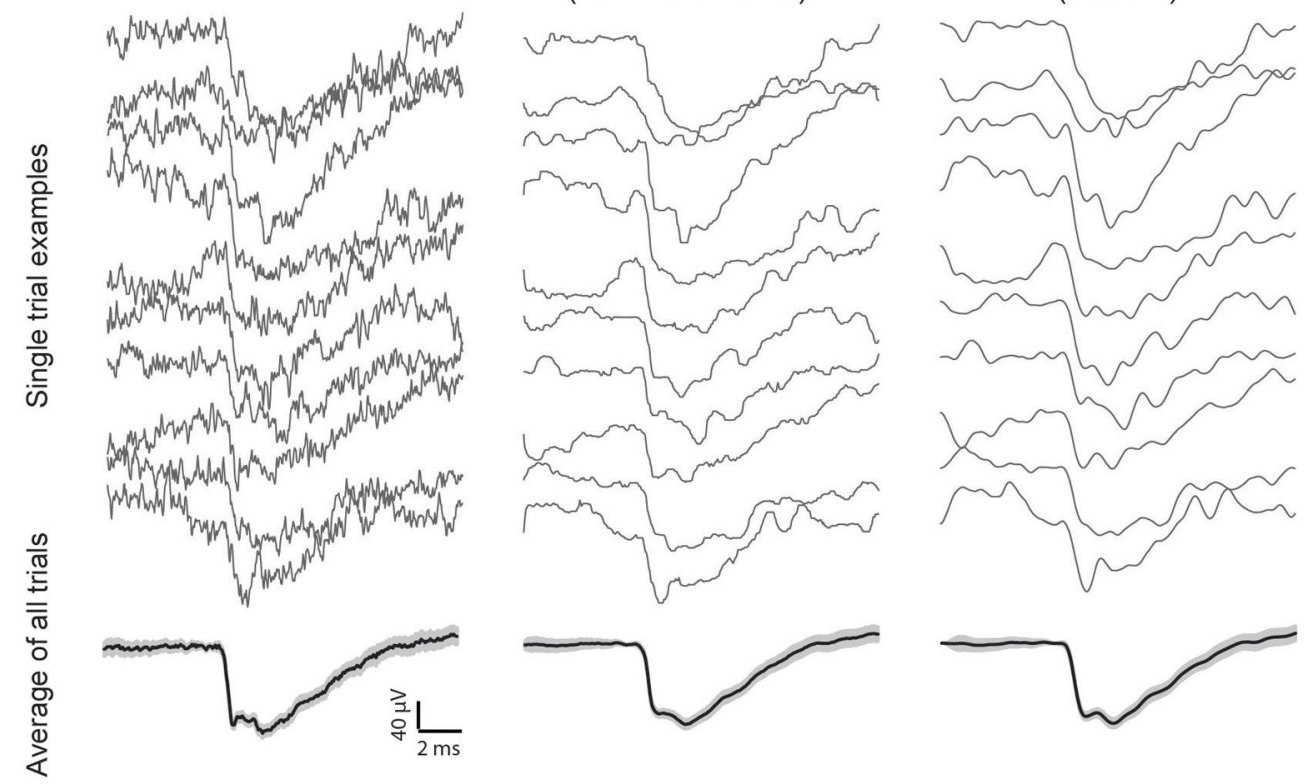

b
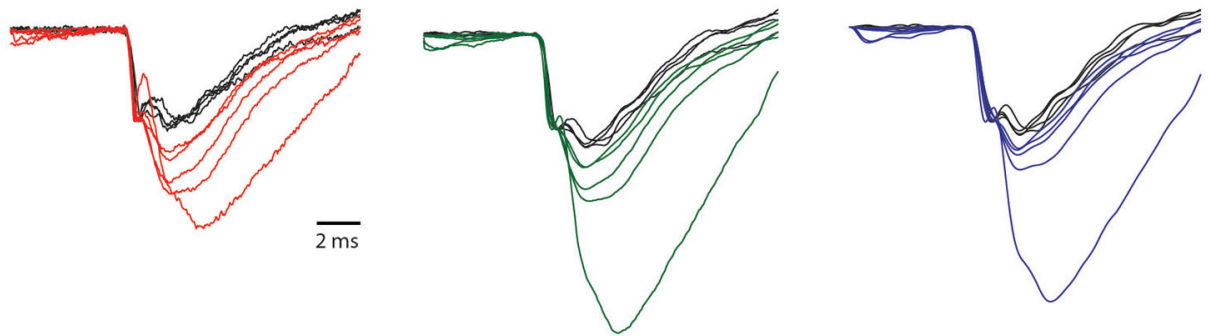

C

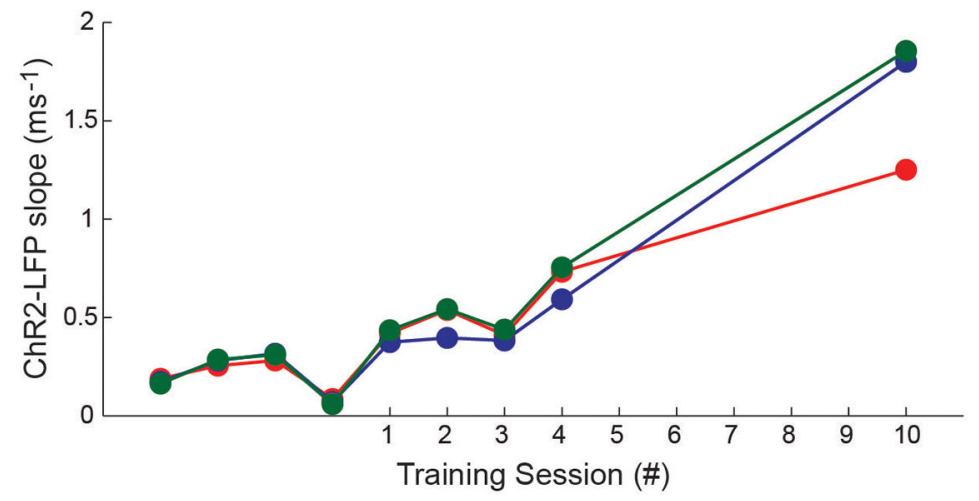

Extended Data 9.

To exclude the possibility that spiking responses affected the ChR2-LFP measurement, we analyzed the data after median or lowpass filtering. (a) Single trial (upper rows) and average (bottom row) examples of unfiltered, median filtered and Butterworth lowpass filtered 
responses. Average traces are presented as mean values (black traces) with 95\% confidence intervals (grey shading). (b) ChR2-LFP examples in Figure 2a with different filter settings. (c) ChR2-LFP measurements from examples shown in Figure 2a at different filter settings.
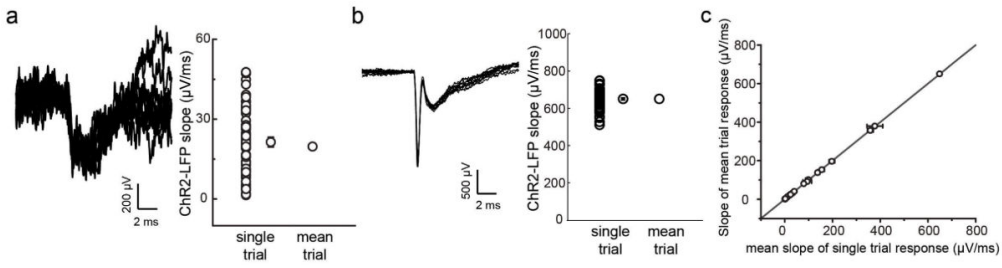

\section{Extended Data 10.}

Changes in ChR2-LFP could result from variation in response timing precision. To rule out this possibility we compared slopes measured from single trial and mean responses. (a) Single trial responses (left panel) and slopes measured from individual trials and mean response (right panel) at a weakly light-responsive site. (b) An example robustly responsive site. (c) Comparison of mean slopes from single trial responses and slopes quantified from mean responses.

\section{Acknowledgments}

We thank B. Burbach for invaluable technical help, R. Eifert for mechanical material support, and J. Cohen for training the rats. AAV-CAGGS-ChR2-Venus was provided by K. Svoboda. We thank U. Livneh and A. Reid for discussions. This work was supported by grants (R01DC012565 and R01NS088649) from the National Institutes of Health (A.M.Z.).

\section{References}

1. Znamenskiy P, Zador AM. Corticostriatal neurons in auditory cortex drive decisions during auditory discrimination. Nature. 2013; 497:482-485.10.1038/nature12077 [PubMed: 23636333]

2. Salzman CD, Britten KH, Newsome WT. Cortical microstimulation influences perceptual judgements of motion direction. Nature. 1990; 346:174-177.10.1038/346174a0 [PubMed: 2366872]

3. Roitman JD, Shadlen MN. Response of neurons in the lateral intraparietal area during a combined visual discrimination reaction time task. The Journal of neuroscience : the official journal of the Society for Neuroscience. 2002; 22:9475-9489. [PubMed: 12417672]

4. Uchida N, Mainen ZF. Speed and accuracy of olfactory discrimination in the rat. Nature neuroscience. 2003; 6:1224-1229.10.1038/nn1142 [PubMed: 14566341]

5. Felsen G, Mainen ZF. Neural substrates of sensory-guided locomotor decisions in the rat superior colliculus. Neuron. 2008; 60:137-148.10.1016/j.neuron.2008.09.019 [PubMed: 18940594]

6. Erlich JC, Bialek M, Brody CD. A cortical substrate for memory-guided orienting in the rat. Neuron. 2011; 72:330-343.10.1016/j.neuron.2011.07.010 [PubMed: 22017991]

7. Raposo D, Sheppard JP, Schrater PR, Churchland AK. Multisensory decision-making in rats and humans. The Journal of neuroscience : the official journal of the Society for Neuroscience. 2012; 32:3726-3735.10.1523/JNEUROSCI.4998-11.2012 [PubMed: 22423093]

8. Brunton BW, Botvinick MM, Brody CD. Rats and humans can optimally accumulate evidence for decision-making. Science. 2013; 340:95-98.10.1126/science.1233912 [PubMed: 23559254]

9. Thompson JA, Felsen G. Activity in mouse pedunculopontine tegmental nucleus reflects action and outcome in a decision-making task. Journal of neurophysiology. 2013; 110:2817-2829.10.1152/jn. 00464.2013 [PubMed: 24089397]

10. Tai LH, Lee AM, Benavidez N, Bonci A, Wilbrecht L. Transient stimulation of distinct subpopulations of striatal neurons mimics changes in action value. Nature neuroscience. 2012; 15:1281-1289.10.1038/nn.3188 [PubMed: 22902719] 
11. Barnes TD, Kubota Y, Hu D, Jin DZ, Graybiel AM. Activity of striatal neurons reflects dynamic encoding and recoding of procedural memories. Nature. 2005; 437:1158-1161.10.1038/ nature04053 [PubMed: 16237445]

12. Schultz W, Dickinson A. Neuronal coding of prediction errors. Annual review of neuroscience. 2000; 23:473-500.10.1146/annurev.neuro.23.1.473

13. Reynolds JN, Hyland BI, Wickens JR. A cellular mechanism of reward-related learning. Nature. 2001; 413:67-70. [pii]. 10.1038/3509256035092560 [PubMed: 11544526]

14. Yin $\mathrm{HH}$, et al. Dynamic reorganization of striatal circuits during the acquisition and consolidation of a skill. Nature neuroscience. 2009; 12:333-341.10.1038/nn.2261 [PubMed: 19198605]

15. Malenka RC, Kocsis JD. Presynaptic actions of carbachol and adenosine on corticostriatal synaptic transmission studied in vitro. The Journal of neuroscience : the official journal of the Society for Neuroscience. 1988; 8:3750-3756. [PubMed: 2848109]

16. Hikosaka O, Takikawa Y, Kawagoe R. Role of the basal ganglia in the control of purposive saccadic eye movements. Physiological reviews. 2000; 80:953-978. [PubMed: 10893428]

17. Carew TJ, Walters ET, Kandel ER. Associative learning in Aplysia: cellular correlates supporting a conditioned fear hypothesis. Science. 1981; 211:501-504. [PubMed: 7455692]

18. Rioult-Pedotti MS, Friedman D, Donoghue JP. Learning-induced LTP in neocortex. Science. 2000; 290:533-536. [PubMed: 11039938]

19. Rumpel S, LeDoux J, Zador A, Malinow R. Postsynaptic receptor trafficking underlying a form of associative learning. Science. 2005; 308:83-88.10.1126/science.1103944 [PubMed: 15746389]

20. Whitlock JR, Heynen AJ, Shuler MG, Bear MF. Learning induces long-term potentiation in the hippocampus. Science. 2006; 313:1093-1097.10.1126/science.1128134 [PubMed: 16931756]

21. Finnerty GT, Roberts LS, Connors BW. Sensory experience modifies the short-term dynamics of neocortical synapses. Nature. 1999; 400:367-371.10.1038/22553 [PubMed: 10432115]

22. Trachtenberg JT, et al. Long-term in vivo imaging of experience-dependent synaptic plasticity in adult cortex. Nature. 2002; 420:788-794.10.1038/nature01273 [PubMed: 12490942]

23. Froemke RC, Merzenich MM, Schreiner CE. A synaptic memory trace for cortical receptive field plasticity. Nature. 2007; 450:425-429.10.1038/nature06289 [PubMed: 18004384]

24. Hofer SB, Mrsic-Flogel TD, Bonhoeffer T, Hubener M. Experience leaves a lasting structural trace in cortical circuits. Nature. 2009; 457:313-317.10.1038/nature07487 [PubMed: 19005470]

25. Fritz J, Shamma S, Elhilali M, Klein D. Rapid task-related plasticity of spectrotemporal receptive fields in primary auditory cortex. Nature neuroscience. 2003; 6:1216-1223.10.1038/nn1141 [PubMed: 14583754]

26. Edeline JM, Weinberger NM. Receptive field plasticity in the auditory cortex during frequency discrimination training: selective retuning independent of task difficulty. Behavioral neuroscience. 1993; 107:82-103. [PubMed: 8447960]

27. Maass W, Natschlager T, Markram H. Real-time computing without stable states: a new framework for neural computation based on perturbations. Neural computation. 2002; 14:25312560.10.1162/089976602760407955 [PubMed: 12433288]

28. Sussillo D, Abbott LF. Generating coherent patterns of activity from chaotic neural networks. Neuron. 2009; 63:544-557.10.1016/j.neuron.2009.07.018 [PubMed: 19709635]

29. Cortes C, Vapnik V. Support-vector networks. Mach Learn. 1995; 20:273-297.10.1007/ BF00994018

30. Hromadka T, Deweese MR, Zador AM. Sparse representation of sounds in the unanesthetized auditory cortex. PLoS biology. 2008; 6:e16.10.1371/journal.pbio.0060016 [PubMed: 18232737] 

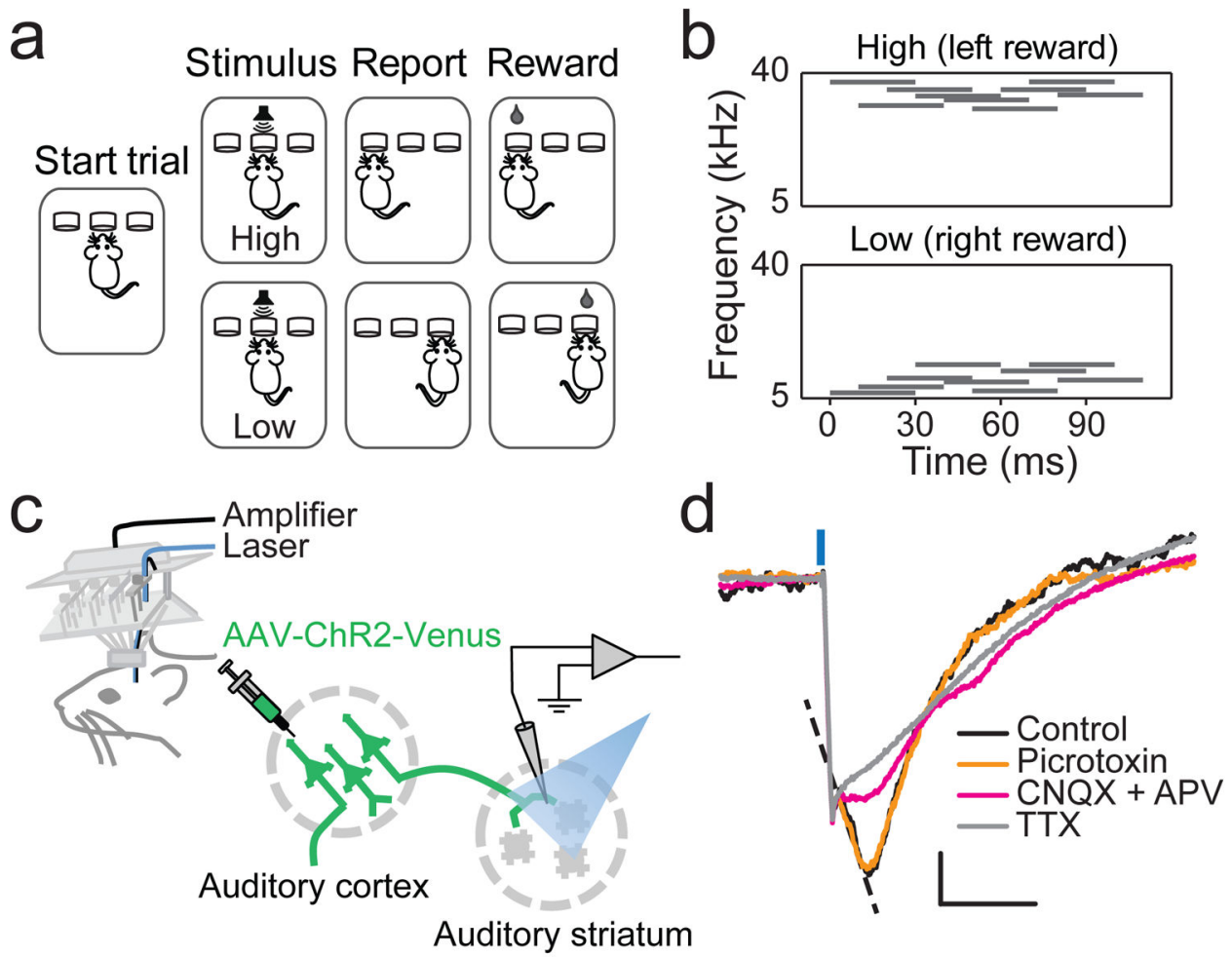

Figure 1. Dissection of ChR2-LFP in vivo

(a) Cloud-of-tones task. (b) Example spectrograms of cloud-of-tones stimuli. (c) Recording paradigm to examine corticostriatal synaptic strength in vivo. (d) ChR2-LFP recorded from auditory striatum under control conditions (black trace) and after application of picrotoxin (orange), CNQX and APV (pink) and tetrodotoxin (light gray). The slope of the CNQX/ APV-sensitive component was used to quantify corticostriatal synaptic strength (dotted line). Scale bars: $20 \mu \mathrm{V}, 5 \mathrm{~ms}$. 


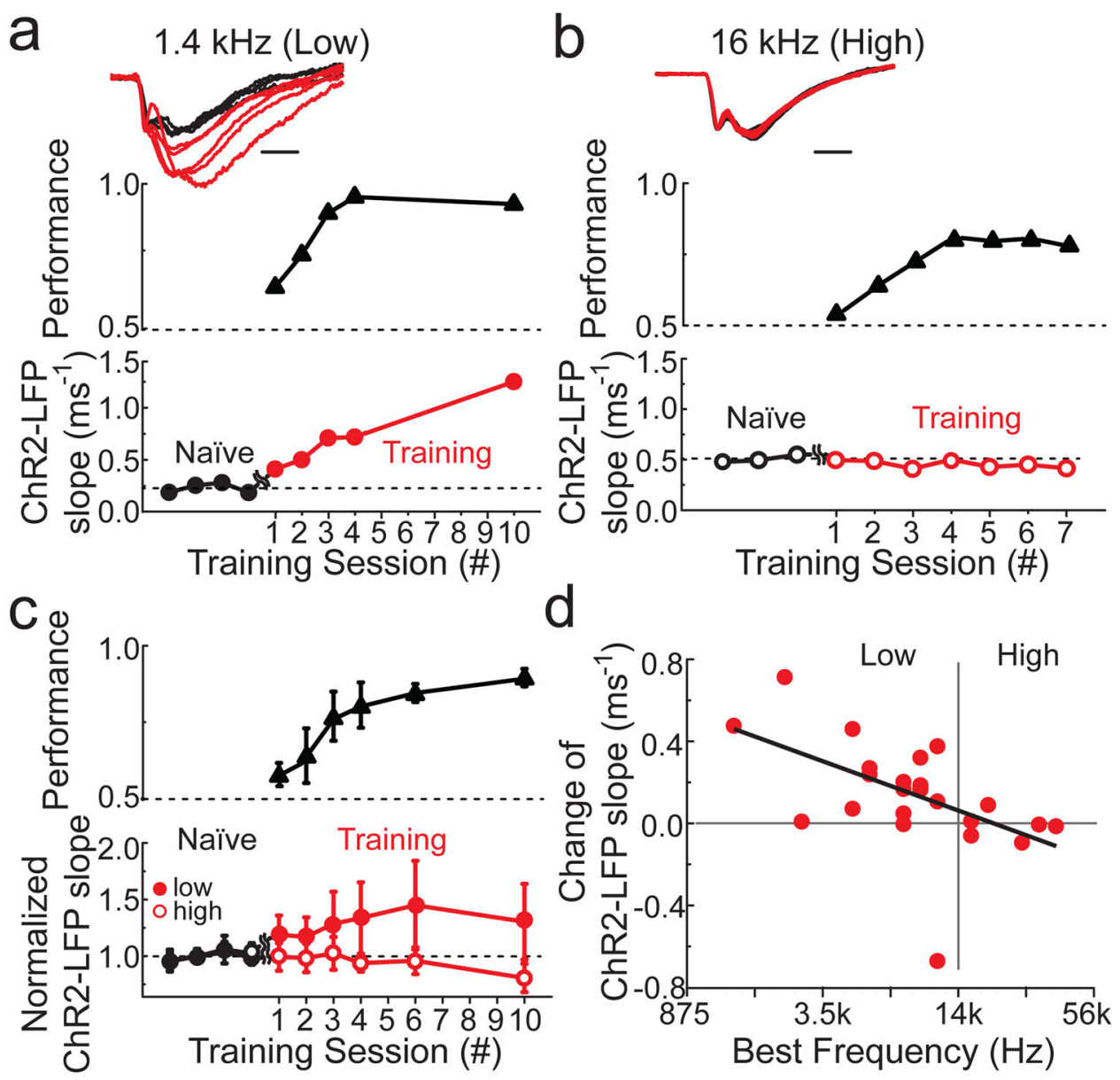

Figure 2. Frequency-selective potentiation of corticostriatal ChR2-LFP slope during learning (a-b) ChR2-LFP (LFP slope: see Methods) before (black) and during (red) training at example sites tuned to low (a) and high frequency (b). Session 1 is defined as the first session in which the animal performed the full task (see Methods). Scale bars: $2 \mathrm{~ms}$. (c) Population average of normalized (see Methods) ChR2-LFP slope during learning for sites tuned to low ( $<14 \mathrm{kHz}, \mathrm{n}=16$ sites, closed circles) and high $(>14 \mathrm{kHz}, \mathrm{n}=6$ sites, open circles) frequencies. Mean \pm s.e.m. (d) Potentiation is restricted to sites tuned to low ( $<14$ $\mathrm{kHz}$ ) frequencies (23 recording sites from 8 rats; least squares regression of potentiation against frequency, $p=0.011$ ). 

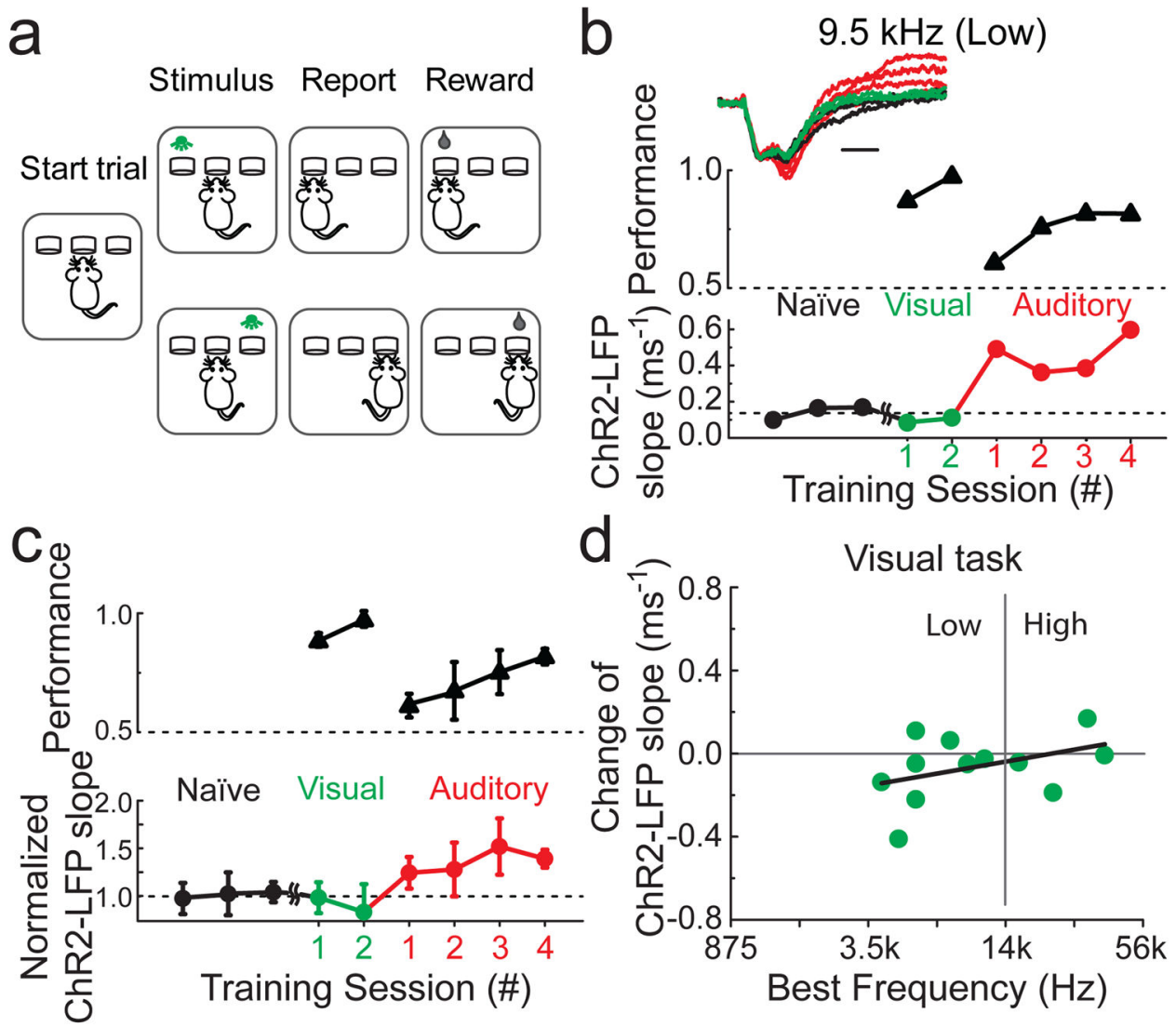

Figure 3. Potentiation of ChR2-LFP slope is modality-specific

(a) Visual 2-AFC task. (b) ChR2-LFP from an example auditory striatum site during visual and auditory task learning, analyzed as in Fig. 2a. Scale bar: $2 \mathrm{~ms}$. (c) Population average of normalized ChR2-LFP slope during visual and auditory task training. (d) Visual training fails to potentiate auditory striatal input (12 recording sites from 4 rats; least squares regression, $p=0.192$ ). 

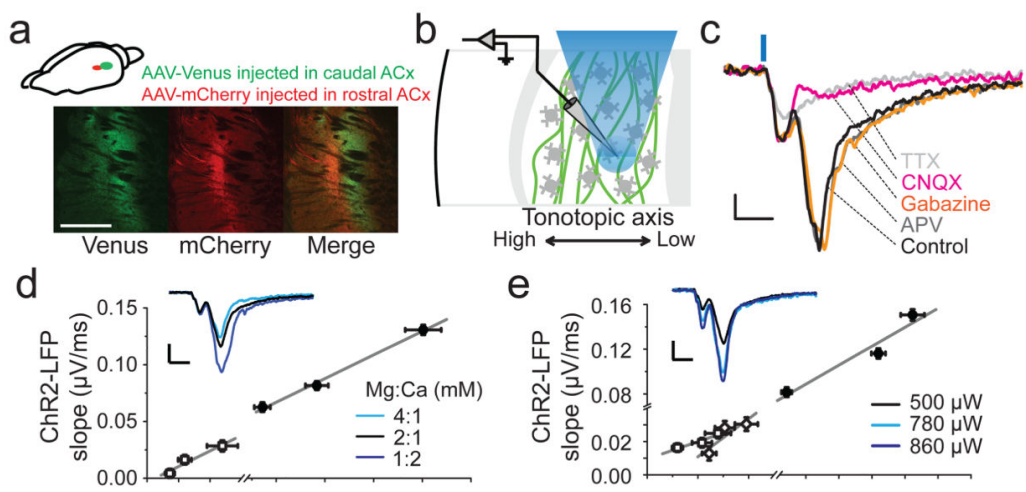

e
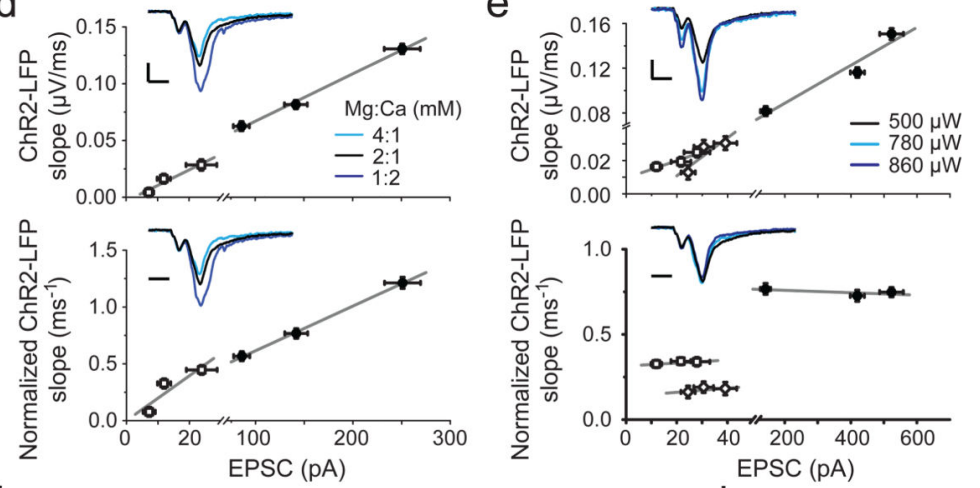

f

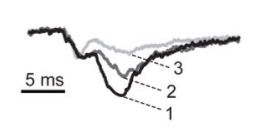

$g$
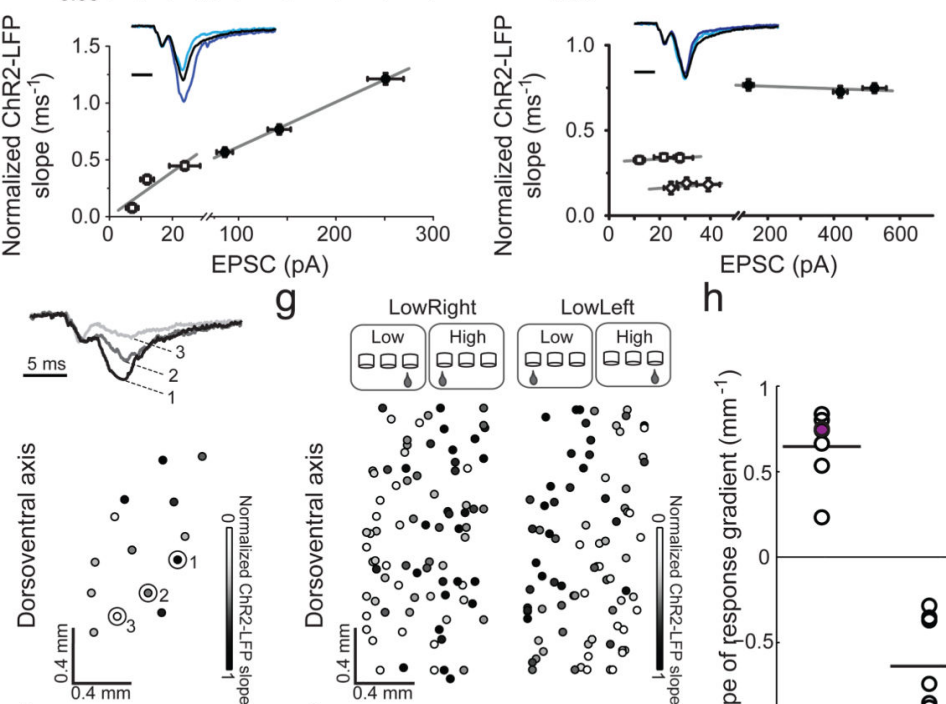

Low High LowLeft

$\mathrm{h}$ $\operatorname{EPSC}(\mathrm{pA})$
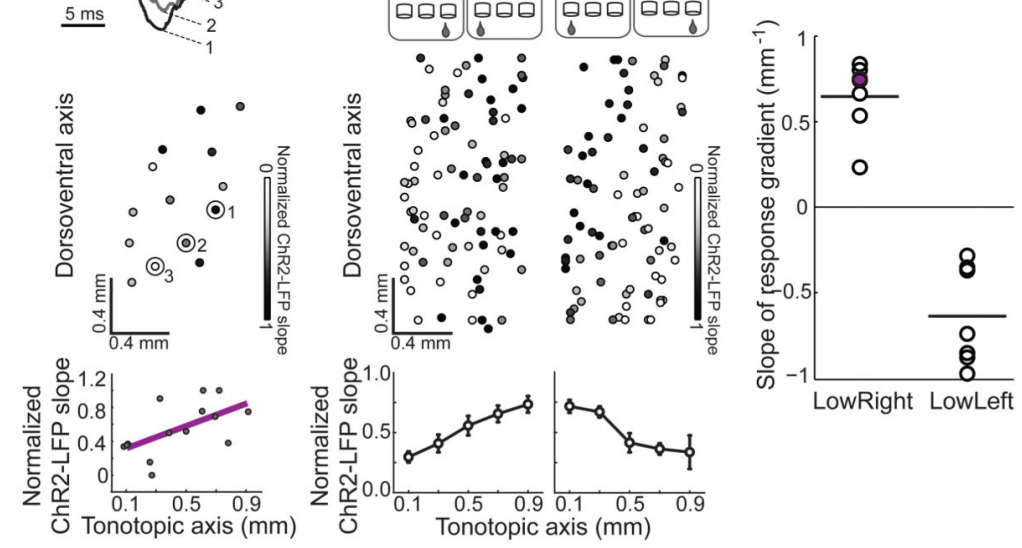

Figure 4. Gradient of corticostriatal ChR2-LFP slopes encodes the association between stimulus and action

(a) Tonotopy of projections from auditory cortex to striatum. Scale bar, $1 \mathrm{~mm}$. (b)

Recording paradigm. Light spot (blue) activates a subset of ChR2-expressing corticostriatal axons (green) near recording site. (c) Pharmacological dissection of ChR2-LFP in a striatal slice. Scale bars, $50 \mu \mathrm{V}$ and $5 \mathrm{~ms}$. (d) Paired in vitro EPSC and LFP at different external divalent ion concentrations. Slopes of LFP measured from raw traces (upper row) and normalized traces (lower row) changed linearly with EPSC amplitudes $\left(R^{2}=0.96 \& 0.99\right.$ for circles, $R^{2}=0.94 \& 0.81$ for squares in upper and lower row respectively. Grey lines in right panels are linear regression fit for each recording pair). Scale bars for $\mathbf{d} \& \mathbf{e}, 100 \mu \mathrm{V}$ and 5 ms. (e) Paired recording at different light levels. Slopes of the LFPs measured from raw traces (upper row) changed monotonically with EPSC amplitudes $\left(\mathrm{R}^{2}=0.94\right.$ for solid circles, $\mathrm{R}^{2}=0.91$ for squares and 0.77 for diamonds). Slopes of the normalized LFPs remain constant (lower row). (f) Normalized ChR2-LFP recorded at many sites within a striatal slice. Sample waveforms (1-3) shown above. ChR2-LFP slope increases with position along tonotopic axis (lower panel). (g) Population data for LowRight ( $\mathrm{n}=7$ rats) and LowLeft ( $\mathrm{n}=7$ 
rats). Error bars are s.e.m. (h) Gradient correctly identifies learned association in 14/14 individual rats (binomial test $p=0.00006$ ). Slope of example shown in $\mathbf{f}$ is indicated in purple. Bars: mean values. 